\title{
Integração entre gestão do conhecimento e business process management: perspectivas de profissionais em BPM
}

\author{
Frederico Cesar Mafra PereiraI \\ http://orcid.org/0000-0002-1971-8069 \\ Ricardo Rodrigues Barbosa ${ }^{I I}$ \\ http://orcid.org/0000-0003-3366-7525 \\ Leonora da Cunha Duarte \\ http://orcid.org/0000-0002-4664-3218 \\ ${ }^{I}$ Centro Universitário UNA, MG, Brasil. \\ Doutor e Mestre em Ciência da Informação (ECI-UFMG.

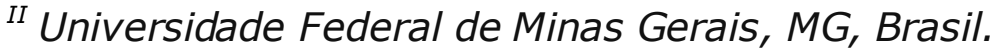 \\ Professor Titular da ECI UFMG. \\ III Fundação Pedro Leopoldo, MG, Brasil. \\ Mestre em Administração pela FPL Educacional.
}

http://dx.doi.org/10.1590/1981-5344/4088

Com o objetivo de identificar de que forma a Gestão do Conhecimento pode contribuir como suporte ao Business Process Management, realizou-se uma pesquisa descritiva, com abordagem quantitativa, do tipo survey. A partir da teoria pesquisada, elaborou-se o modelo conceitual, base para o instrumento de coleta de dados, que investigou a relação integrativa entre as práticas do Modelo SECI e as atividades do cido de vida da gestão por processos. Verificou-se que há o suporte da criação do conhecimento em todas as fases do ciclo de vida e que - Modelo SECI impacta de forma diferente nessas atividades. Foram também levantadas as principais práticas de conversão do conhecimento nas perspectivas dos respondentes. O estudo amplia a discussão sobre a 
relação integrativa entre os temas pesquisados, preenchendo uma lacuna entre a teoria sobre a conversão do conhecimento e as práticas aplicáveis nas atividades de gestão por processos; além de contribuir para a realização de pesquisas futuras sobre o tema, a partir do modelo conceitual desenvolvido, com 0 apoio do instrumento de coleta de dados na íntegra ou modificado; os resultados da pesquisa também podem servir de apoio a gestores no tocante à priorização ao estímulo de determinadas práticas de conversão do conhecimento.

Palavras-chave: Gestão do Conhecimento. Gerenciamento de Processos de Negócio. Modelo SECI. Ciclo de Vida BPM CBOK.

\section{Integration between knowledge management and business process management: perspectives of BPM professionals}

In order to identify how Knowledge Management can contribute to support the Business Process Management, a descriptive survey with quantitative approach was conducted. From the researched theory, a conceptual model, which was the basis for the data collection instrument, was elaborated. It was used to investigate the integrative relationship between the practices of the SECI Model and the activities of the process management life cycle. It was found that knowledge creation is supported at all stages of the life cycle and that the SECI Model impacts differently on these activities. The main practices of knowledge conversion from the respondents' perspectives were also raised. he study broadens the discussion about the integrative relationship between the researched themes, filling a gap between the theory of knowledge conversion and the applicable practices in process management activities; In addition to contributing to future research on the theme, based on the developed conceptual model, with the support of the 
data collection instrument in its entirety or modified version; the survey results can also support managers in prioritizing the stimulation of certain knowledge conversion practices.

Keywords: Knowledge Management. Business Process Management. SECI Model. CBOK Lifecycle.

Recebido em 18.08.2019 Aceito em 09.12.2020

\section{Introdução}

A gestão por processos, como prática administrativa, tem o objetivo de assegurar a melhoria contínua do desempenho da organização, por meio da elevação dos níveis de qualidade de seus processos de negócios. Neste contexto, práticas de Gestão do Conhecimento (GC), aplicadas à operação e à gestão de processos, constituem um recurso com grande potencial para a identificação de problemas e oportunidades inerentes aos processos e, por consequência, transformam-se em um importante facilitador para a melhoria contínua das operações (CONTADOR et al., 2005). Tal posicionamento é apoiado por Van der Aalstet al. (2016) ao afirmarem que o pensamento por processos se tornou comum na maioria das organizações e que, em função disso, precisa ser apoiado por práticas de GC.

O Business Process Management (BPM), ou gerenciamento de processos de negócio, tem primordial papel no mundo empresarial porque faz com que a organização opere a partir de todas as dimensões do seu negócio. O objetivo é viabilizar a melhor coordenação e integração dos trabalhos. Com isso, habilita-se o foco direcionado a clientes, tornando possível que a organização se prepare antecipadamente para mudanças. O BPM provê uma visão sistêmica da organização, com um melhor entendimento dos seus processos de negócio, isto é, da sua cadeia de valor (BALDAM; VALLE; ROZENFELD, 2014).

Para Moreno e Santos (2012), a gestão organizacionaldeixa de gerar os benefícios esperados quando há uma distância entre os processos de negócio e os processos de conhecimento. A efetividade organizacional está condicionada, na concepção dos autores, à aplicação do conhecimento na melhoria e na transformação de processos de negócio. Neste contexto, Davenport (2013) assegura que melhorar o desempenho dos trabalhadores do conhecimento é bastante desafiador e carece de ferramentas e metodologias adequadas. Portanto, é viável o questionamento se o BPM pode contribuir para tão árdua tarefa, posto que 
"uma maneira consagrada pelo tempo de melhorar qualquer forma de trabalho é tratá-lo como um processo" (p.18).

A integração entre GC e BPM é estratégica, portanto, porque os processos de negócio agem como elemento integrador entre os ativos institucionais, dentre eles o conhecimento organizacional. A GC orientada por processos de negócio capacita a concentração da equipe no que é essencial, ou seja, na cadeia de valor organizacional (SORDI, 2018). Pesquisas que busquem desvelar os processos de negócio e sua gestão têm grande importância para fornecer o contexto prático das atividades de GC (MARJANOVIC; FREEZE, 2012).

Nesse sentido, o objetivo geral desta pesquisa foi identificar de que forma a GC pode contribuir como suporte ao BPM, na percepção de profissionais que atuam com gerenciamento de processos de negócio. Para tal, foi realizada uma pesquisa descritiva, com abordagem quantitativa, do tipo survey. A partir do referencial teórico pesquisado, elaborou-se o modelo conceitual da pesquisa referente à relação integrativa entre as práticas de GC do Modelo SECI e as atividades de BPM. A coleta de dados ocorreu por meio de um questionárioautoaplicado, disponibilizado online. Os dados levantados foram analisados por meio da estatística descritiva (para a organização, sumarização e descrição do conjunto dos dados) e da estatística inferencial (para a obtenção de informações sobre a população alvo a partir dos resultados observados).

\section{Referencial Teórico}

Esta seção apresenta os principais aspectos da fundamentaçãoque serviu de base para o modelo conceitual, utilizado na elaboração do instrumento de coleta de dados. Os pilares do arcabouço teórico são a Gestão do Conhecimento (principais conceitos e visão geral do Modelo SECI), o Business ProcessManagement (definições mais relevantes e o ciclo de vida das atividades de BPM) e a integração entre GC e BPM (com o quadro resumo das práticas de GC indicadas na literatura para cada fase do ciclo de vida de BPM).

\subsection{Gestão do Conhecimento (GC)}

Nonaka e Takeuchi (1997) sustentam que, para lidarem com as mudanças no ambiente de maneira dinâmica, as organizações precisam criar informação e conhecimento e não apenas processá-los de forma eficiente. Para Drucker (2000), organizações baseadas em conhecimento precisam de propósitos claros e comuns que possam ser traduzidos em ações específicas. Portanto, apesar de ser desafiador o gerenciamento do conhecimento organizacional, quando há a criação do conhecimento e seu adequado uso, observa-se um impacto direto na melhoria da 
produtividade da empresa e na acumulação de riqueza (YAXIONGet al., 2008).

O conhecimento também é apontado como vantagem competitiva por Bem e Ribeiro Júnior (2006). Os autores afirmam que o conhecimento só aumenta quando é utilizado, dividido ou compartilhado, podendo ser considerado um recurso infinito, que pode trazer grandes benefícios, principalmente no longo prazo. O desafio é justamente o seu gerenciamento, posto que tal processo tem um caráter interdisciplinar, envolvendo áreas de conhecimento como organização, tecnologias de informação, comunicação, dentre outras, e profissionais de diversos perfis. Portanto, para ser efetiva, a GC tem que ser articulada.

Davenport e Prusak (1998, p.30) consideram a GC como uma "reunião de todas as tarefas que envolvam a geração, codificação e transferência do conhecimento". Já para Sabbag (2007, p.60), a GC é "um sistema integrado que visa desenvolver conhecimento e competência coletiva para ampliar o capital intelectual de organizações e a sabedoria das pessoas". Na conceituação de Robbins (1999), o processo da GC começa com a identificação de conhecimentos importantes para a organização, seguida do desenvolvimento de redes (de preferência informatizadas) e de sistemas de informação, que tornem tais conhecimentos rapidamente disponíveis para aqueles que deles necessitam.

Para ser exitosa, a GC deve estar intimamente vinculada processos de interesse crítico para a empresa. É preciso haver meios para organizar informações e conhecimentos, conforme cada tarefa do fluxo de tais processos. Ou seja, os processos de negócio precisam estar habilitados pelo conhecimento (RECORDS, 2005). Mafra Pereira (2016) concorda com a fluidez do processo de criação de conhecimento e destaca, também, o importante papel do uso de fontes de informação pelos tomadores de decisão com vistas à criação do conhecimento organizacional. Para esteautor, o conhecimento organizacional é um processo iniciado no nível individual e que se expande de forma interativa pela organização.

A construção de metodologias de GC, baseadas em estudos e pesquisas a partir de experiências vivenciadas por várias organizações, originam os denominados "modelos de GC". Há inúmeros modelos criados com base nesta lógica, mas, nesta pesquisa, adotou-se o modelo proposto por Nonaka e Takeuchi (1997), denominado 'Modelo SECI'. Tal modelo se baseia no fato de que o conhecimento humano é criado e expandido através de interação social entre o conhecimento tácito e o explícito. Tal conversão do conhecimento ocorre pelas ações: socializar (S), externalizar (E), combinar (C) e internalizar (I). Na socialização, ocorre a troca de conhecimentos tácitos. A externalização se dá quando o conhecimento tácito é expresso e codificado, transformando-se em conhecimento 
explícito. Ocorre a combinação quando novos e antigos conhecimentos explícitos são combinados e transferidos. Na internalização, os novos conhecimentos explícitos são apropriados pelo indivíduo, transformandose em conhecimento tácito. Esse processo de criação de conhecimento é composto por cinco fases: o compartilhamento de conhecimento tácito; a criação de conceitos; a justificação dos conceitos; a construção de um arquétipo ou modelo; e a difusão interativa do conhecimento.

\subsection{Business Process Management (BPM)}

Em décadas recentes, teorias e práticas administrativas trouxeram contribuições importantes para o desenvolvimento empresarial e para o atendimento de novas demandas de mercado, com destaque para a introdução do conceito de processos de negócio como forma de gerenciar e estruturar as empresas. Assim, a gestão por processos tem se tornado um fator crucial para o sucesso e o desenvolvimento das empresas (HRASTNIKet al., 2007).

Especificamente sobre a caracterização do BPM, Silva (2017) afirma que se trata de um movimento evolutivo da gestão de processos organizacionais em que a tecnologia e a automatização são pontos importantes. A mudança é fundamental e visa à intensificação da necessidade do gerenciamento dos processos end-to-end (ponta a ponta). O foco é a obtenção de resultados, agilidade e adaptabilidade. Como a tecnologia tem fundamental papel, surgem os denominados Business Process Management Systems (BPMS), que são os sistemas informatizados de gestão dos processos de negócio, específicos para a metodologia BPM.

O BPM tem se tornado uma disciplina madura, com um conjunto bem estabelecido de princípios, métodos e ferramentas que combinam conhecimento da tecnologia da informação, ciências da gestão e engenharia industrial. O objetivo da metodologia BPM é melhorar processos de negócios (VAN DER AALSTet al., 2016).

Em relação aos conceitos basilares do tema, vale ressaltar a definição e a tipologia do termo "processo" constantes no Common BodyofKnowledge (CBOK) da Associationof Business Process Management Professionals (ABPMP). "Processo é uma agregação de atividades e comportamentos executados por humanos ou máquinas para alcançar um ou mais resultados" (ABPMP, 2015, p.46). Tal conceituação é relevante por incorporar a execução das atividades, seja pelo homem ou por equipamentos por ele criados ou operados. Ainda de acordo com o CBOK, o processo de negócio pode ser classificado em três tipos: primário, de suporte e de gerenciamento. A compreensão da interação dos diferentes tipos de processos de negócio é essencial para sua gestão. Os processos primários formam a chamada cadeia de valor da organização (que é a 
representação das suas atividades essenciais). Os processos de suporte proveem o apoio aos processos primários, mas podem também ser fundamentais e estratégicos, na medida em que aumentam a capacidade de realização dos processos essenciais. Os processos de gerenciamento asseguram que os demais processos sejam executados de forma a se obter os resultados pretendidos pela organização (ABPMP, 2015).

Uma contribuição de grande relevância para os profissionais que adotam o gerenciamento de processos de negócio é a possiblidade de contar com uma estrutura básica de atividades de trabalho, ou seja, um ciclo de atividades. Esse ciclo fornece subsídios que permitem aos tomadores de decisões saber por onde iniciar e como guiar os trabalhos de BPM. No entanto, somente olhando para um modelo esquematizado, é desafiador prever se ele será ou não efetivo, posto que a forma como o mesmo será implantado faz toda a diferença (BALDAMet al., 2014).

Em função do significativo volume de modelos ou ciclos de BPM disponíveis em estudos e no mercado, nesta pesquisa optou-se por pela adoção do ciclo de vida de BPM proposto no CBOK, por este ser de conhecimento comum entre os participantes da pesquisa, como será demonstrado na caracterização da população. De acordo com o referido corpo de conhecimento comum, a prática do gerenciamento de processos de negócio envolve um conjunto de atividades próprias, realizadas e retroalimentadas ciclicamente, isto é, um ciclo de vida BPM típico, focado no gerenciamento da mudança, que envolve: planejar, analisar, desenhar, implementar, monitorar / controlar e refinar. Conforme o CBOK, "BPM implica um comprometimento permanente e contínuo da organização para o gerenciamento de seus processos" (ABPMP, 2015, p.63).

\subsection{Integração entre GC e BPM}

Apesar de terem evoluído independentemente, os ciclos de vida de GC e BPM deixam claro que o conhecimento é um atributo inerente a processos de negócio e, por isso, "uma abordagem complementar e integrada das duas áreas de conhecimento é possível e desejável" (SENA, 2015, p.201). Tanto que Jung, Choi e Song (2007) ressaltam que há várias pesquisas científicas cujo foco é introduzir o conceito de processo em GC ou o conceito de conhecimento em BPM, com o objetivo de combinar as vantagens dos dois paradigmas.

A partir do levantamento das atividades inerentes a cada fase do ciclo de vida BPM proposto pelo CBOK, em comparação com práticas de gestão do conhecimento sugeridas por diversos autores de acordo com a conversão do conhecimento do Modelo SECI, estruturou-se a Figura $1 \mathrm{com}$ as principais contribuições do referencial teórico da pesquisa completa que serviram de base para o modelo conceitual do instrumento de pesquisa aplicado. 
Integração entre gestão do conhecimento e business process management: perspectivas de profissionais em bpm
Frederico Cesar Mafra Pereira; Ricardo Rodrigues Barbosa; Leonora da Cunha Duarte

Figura 1: Principais contribuições do RT para a pesquisa

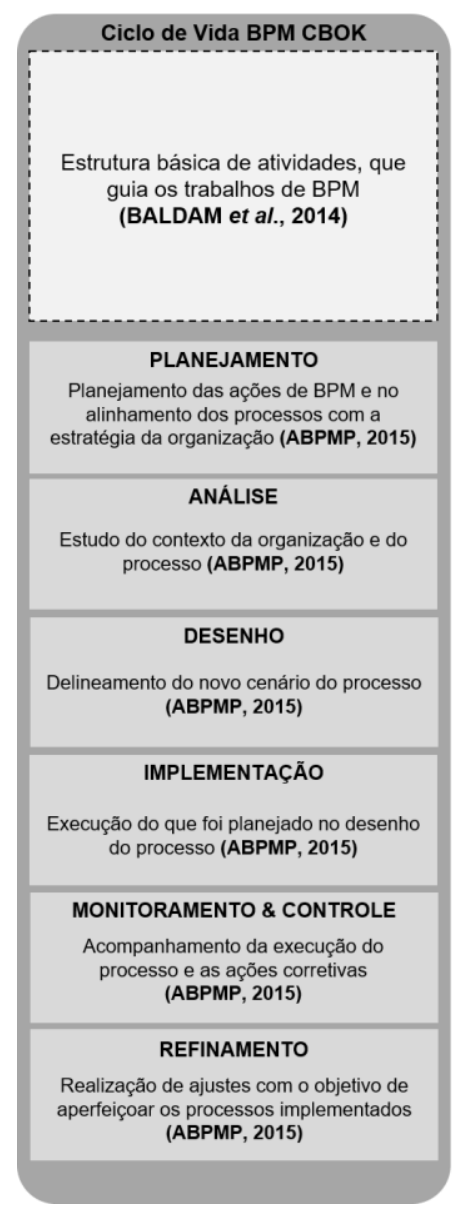

\begin{tabular}{|c|c|c|c|}
\hline \multicolumn{4}{|c|}{ Modelo SECI } \\
\hline $\begin{array}{r}\text { Força motriz da cria } \\
\text { Espiral do conhecim } \\
\text { Fases: compartilhame } \\
\text { construção de }\end{array}$ & $\begin{array}{l}\text { o do conhecimento: mot } \\
\text { to apoiada por condiçõe } \\
\text { o do conhecimento tácitc } \\
\text { arquétipo; nivelação do }\end{array}$ & $\begin{array}{l}\text { zação e conversão do co } \\
\text { romotoras e por um cont } \\
\text { criação dos conceitos; jus } \\
\text { onhecimento. (Takeuchi } 8\end{array}$ & $\begin{array}{l}\text { hecimento (modos). } \\
\text { exto capacitante "ba". } \\
\text { ificação dos conceitos; } \\
\text { Nonaka, 2008) }\end{array}$ \\
\hline $\begin{array}{l}\text { SOCIALIZAÇÃO } \\
\text { Experiência direta p/ criar } \\
\text { conhecimento (tácito p/ } \\
\text { tácito) (TAKEUCHI; } \\
\text { NONAKA, 2008) }\end{array}$ & $\begin{array}{l}\text { EXTERNALIZAÇÃO } \\
\text { Diálogo e reflexão p/ } \\
\text { articular o conhecimento } \\
\text { (tácito p/ explicito) } \\
\text { (TAKEUCHI; NONAKA, } \\
\text { 2008) }\end{array}$ & $\begin{array}{l}\text { COMBINAÇÃO } \\
\text { Sistematizaçāo e aplicação } \\
\text { do conhecimento e da } \\
\text { informação (explicito p/ } \\
\text { explicito) (TAKEUCHI; } \\
\text { NONAKA, 2008) }\end{array}$ & $\begin{array}{l}\text { INTERNALIZAÇĀO } \\
\text { Aprendizado e aquisiçāo de } \\
\text { novo conhecimento na } \\
\text { prática (explicito p/ tácito) } \\
\text { (TAKEUCHI; NONAKA, } \\
\text { 2008) }\end{array}$ \\
\hline $\begin{array}{l}\text { Reuniōes abertas e projetos } \\
\text { cooperativos entre diretorias } \\
\text { (TAKEUCHI; NONAKA, 2008) }\end{array}$ & $\begin{array}{l}\text { Captura de conhecimento de } \\
\text { especialistas e o uso de } \\
\text { sistemas de suporte à decisāo } \\
\text { (BECERRA-FERNANDEZ; } \\
\text { SABHERWAL, 2001) }\end{array}$ & $\begin{array}{c}\text { Visão corporativa (TAKEUCHI; } \\
\text { NONAKA, 2008) Repositório de } \\
\text { informaçöes, melhores práticas } \\
\text { eliçeses aprendidas } \\
\text { (BECCERRA-FERNANDEZ; } \\
\text { SABHERWAL, 2001) }\end{array}$ & $\begin{array}{c}\text { Reuniões presenciais } \\
\text { (BECERRA-FERNANDEZ; } \\
\text { SABHERWAL, 2001) } \\
\text { Práticas e relatos orais } \\
\text { (TAKEUCHI; NONAKA, 2008) }\end{array}$ \\
\hline $\begin{array}{l}\text { Reuniōes informais e a } \\
\text { observaçăo participante } \\
\text { (CHOO, 2006) }\end{array}$ & $\begin{array}{l}\text { Metáforas e analogias } \\
\text { (TAKEUCHI; NONAKA, } \\
\text { 2008; NONAKA2000) }\end{array}$ & $\begin{array}{l}\text { Consulta à base de dados } \\
\text { (TAKEUCHI; NONAKA, 2008) } \\
\text { Repositório de informaçōes, } \\
\text { melhores práticas e liç̄es } \\
\text { (BECERRRAFERNANDEZ; } \\
\text { SABHERWAL, 2001) }\end{array}$ & $\begin{array}{c}\text { Documentos } \\
\text { (TAKEUCHI; NONAKA, 2008) } \\
\text { Observaçăo da prática } \\
\text { (BECERRA-FERNANDEZ; } \\
\text { SABHERWAL, 2001) }\end{array}$ \\
\hline $\begin{array}{c}\text { Sessōes de discussão dos } \\
\text { processos } \\
\text { (OLIVEIRA et al., 2010) }\end{array}$ & $\begin{array}{l}\text { Metáforas e analogias para a } \\
\text { proposição de um modelo real } \\
\text { do processo (NONAKA, 2000) }\end{array}$ & $\begin{array}{c}\text { Uso de BPMN (OLIVEIRA et } \\
\text { al., 2010) } \\
\text { Consulta à base de dados da } \\
\text { modelagem de processos } \\
\text { (TAKEUCHI; NONAKA, } \\
\text { 2008) }\end{array}$ & $\begin{array}{c}\text { Simulaçöes e interpretaçăo } \\
\text { de papéis para a vivência do } \\
\text { processo } \\
\text { (CHOO, 2006) }\end{array}$ \\
\hline $\begin{array}{c}\text { Relacionamento } \\
\text { aprendiz/mestre e o } \\
\text { treinamento no trabalho } \\
\text { (TAKEUCHI; NONAKA, } \\
\text { 2008) }\end{array}$ & $\begin{array}{l}\text { Histórias de experiências e } \\
\text { compartihamento de } \\
\text { vivências (CHOO, 2006) }\end{array}$ & $\begin{array}{l}\text { Uso de BPMN (OLIVEIRA et } \\
\text { al., 2010) Repositório de } \\
\text { informaç̄os, melhores } \\
\text { práticas e liç̄os aprendidas } \\
\text { (BECERRA-FERNANDEZ; } \\
\text { SABHERWAL, 2001) }\end{array}$ & $\begin{array}{l}\text { Manuais } \\
\text { (TAKEUCHI; NONAKA, 2008) } \\
\text { Treinamento no posto de trabalho } \\
\text { e aprendizado na prática } \\
\text { (BECERRA-FERNANDEZ; } \\
\text { SABHERWAL, 2001) }\end{array}$ \\
\hline $\begin{array}{l}\text { Sessōes de discussão dos } \\
\text { processos (OLIVEIRA et al., } \\
\text { 2010) Rotaçăo de funcionários } \\
\text { (BECERRA-FERNANDEZ; } \\
\text { SABHERWAL, 2001) }\end{array}$ & $\begin{array}{c}\text { Explicitação clara de regras e } \\
\text { fluxos } \\
\text { (OLIVEIRA et al., 2010) }\end{array}$ & $\begin{array}{c}\text { Uso de BPMS } \\
\text { (OLIVEIRA et al., 2010) } \\
\text { Troca de relatórios e } \\
\text { memorandos (CHOO, 2006) }\end{array}$ & $\begin{array}{c}\text { Documentos } \\
\text { (TAKEUCHI; NONAKA, 2008) } \\
\text { Aprendizado por observaçăo } \\
\text { (BECERRA-FERNANDEZ; } \\
\text { SABHERWAL, 2001) }\end{array}$ \\
\hline $\begin{array}{c}\text { Reuniōes informais } \\
\text { (TAKEUCHI; NONAKA, 2008) } \\
\text { Retiros (BECERRA- } \\
\text { FERNANDEZ; SABHERWAL, } \\
\text { 2001) Comunidades de práticas } \\
\text { e grupos sociais (CHOO, 2006) }\end{array}$ & $\begin{array}{l}\text { Bases de dados e } \\
\text { ferramentas de colaboração } \\
\text { (BECERRA-FERNANDEZ; } \\
\text { SABHERWAL, 2001) }\end{array}$ & $\begin{array}{l}\text { Visão corporativa (TAKEUCHI; } \\
\text { NONAKA, 2008) Repositório } \\
\text { de informaçōes, melhores } \\
\text { práticas e liçōes aprendidas } \\
\text { (BECERRAAFERNANDEZ; } \\
\text { SABHERWAL, 2001) }\end{array}$ & $\begin{array}{l}\text { Reuniōes presenciais } \\
\text { (BECERRA-FERNANDEZ; } \\
\text { SABHERWAL, 2001) } \\
\text { Histórias (CHOO, 2006) } \\
\text { Relatos orais (TAKEUCH; } \\
\text { NONAKA, 2008) }\end{array}$ \\
\hline
\end{tabular}

Fonte: Adaptado de Nonaka (2000); Becerra-Fernandez;Sabherwal (2001); Choo (2006); Takeuchi; Nonaka (2008); Oliveira et al. (2010); Baldam et al. (2014); ABPMP (2015).

Com base nas principais contribuições do referencial teórico, foi estruturado, portanto, um diagrama da pesquisa (Figura 2) para representar a relação integrativa entre GC e BPM investigada na pesquisa de campo.

Figura 2: Diagrama da pesquisa 


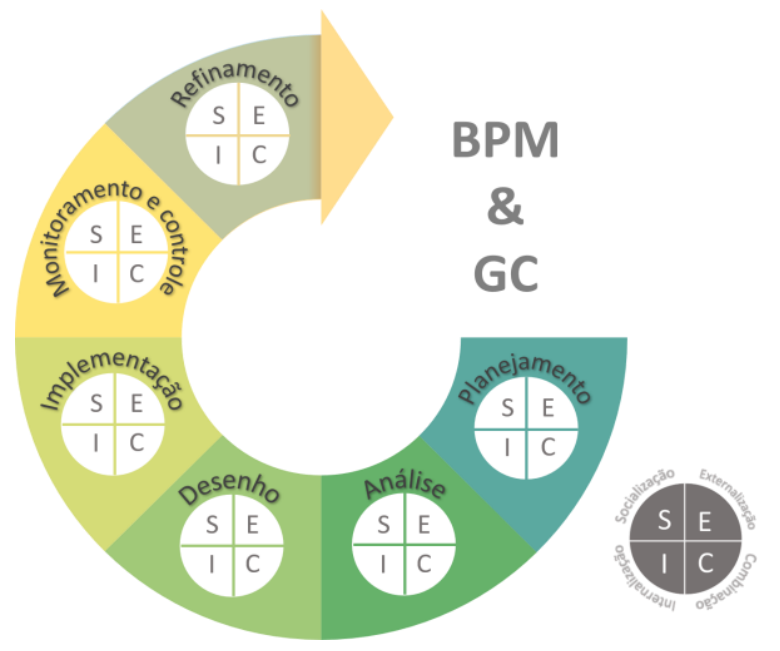

Fonte: Elaborado pelos autores (2019)

\section{Procedimentos Metodológicos}

Neste estudo, optou-se pela pesquisa descritiva, com abordagem quantitativa. SegundoMalhotra (2012), a pesquisa descritiva é um estudo conclusivo que busca descrever algo, e Oliveira (2001) esclarece que a pesquisa quantitativa visa determinar a quantidade de opiniões e dados, oriundos da coleta de informação, além de utilizar técnicas estatísticas. O conhecimento gerado a partir desse tipo de pesquisa pode ser estendido, com determinada margem de erro, para toda a população cuja amostra foi estudada (VIEIRA, 2009).

Como técnica de coleta e análise de dados, adotou-se o método de levantamento (enquete) ou também chamado pesquisa de survey. Segundo Freitaset al. (2000), seu objetivo é produzir descrições quantitativas de uma população, fazendo uso de um instrumento predefinido, o qual, neste estudo, foi um questionário disponibilizado online.

A população alvo desta pesquisa foi de 3.300 profissionais, assim distribuídos:

- 887 profissionais certificados em BPM pela ABPMP Brasil - Certified Business ProcessProfessionals (CBPPs) -, cujos emails estavam disponíveis no site da associação à época do envio dos convites;

- 2.500 profissionais participantes de eventos realizados pela Gestão Regional Minas da ABPMP Brasil para a divulgação de práticas de BPM no Estado. Todavia, como os eventos ocorreram em Minas Gerais, foram desconsiderados, da rede de contatos da regional, os 87 CBPPs mineiros, por já estarem contabilizados no item anterior.

A partir dessa população, considerando um nível de confiança estatística de $95 \%$, com margem de erro de $5 \%$, calculou-se que seria 
necessária uma amostra de 345 respondentes para a validade da análise estatística dos resultados.

Para identificar de que forma a GC pode contribuir como suporte ao BPM, na percepção de profissionais que atuam com gerenciamento de processos de negócio, o instrumento de pesquisa foi desenvolvido considerando as fases do Ciclo de Vida BPM CBOK (planejamento, análise, desenho, implementação, monitoramento \& controle e refinamento) e o apoio do Modelo SECI (e práticas de GC) na execução das atividades de BPM. A estrutura do instrumento, portanto, foi definida com vistas a apurar quais ferramentas são, na percepção dos respondentes, mais aplicadas na prática, considerando-se as seguintes dimensões para a estruturação da pesquisa: a) Modelo SECI (_M) como apoiador do ciclo de vida BPM CBOK que engloba as fases: planejamento, análise, desenho, implementação, monitoramento / controle e refinamento; b) Práticas de socialização (_S) como apoiadoras em cada fase do ciclo; c) Práticas de externalização (_E) como apoiadoras em cada fase do ciclo; d) Práticas de combinação (_C) como apoiadoras em cada fase do ciclo; e) Práticas de internalização (_I) como apoiadoras em cada fase do ciclo.

A Figura 3 apresenta o modelo conceitual de pesquisa que contempla, além das teorias dos autores pesquisados no referencial teórico, a representação das relações entre os conceitos e práticas do Modelo SECI e as atividades do Ciclo de Vida BPM CBOK. 
Figura 3: Modelo Conceitual da pesquisa

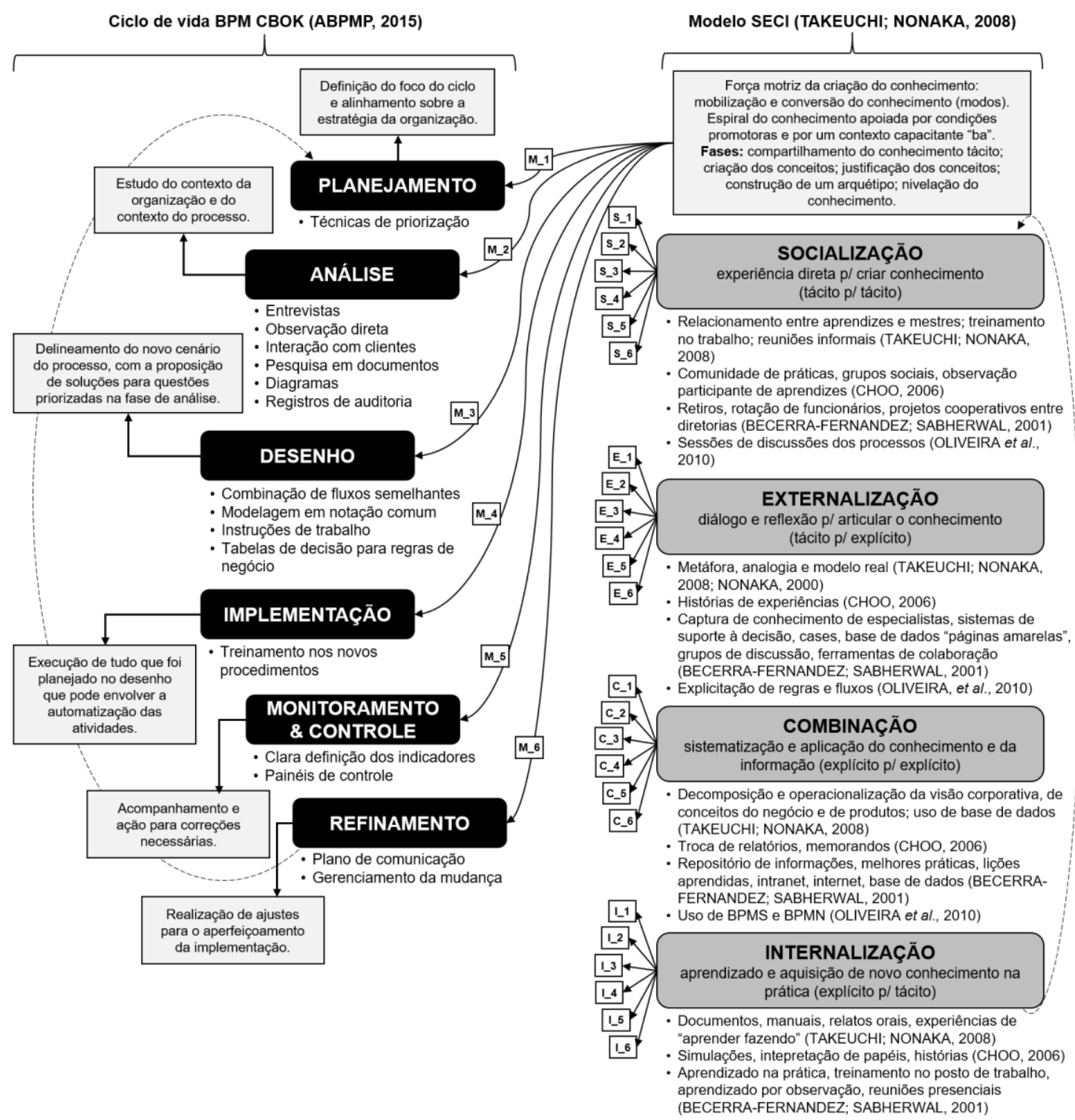

Fonte: Adaptado de Nonaka (2000); Becerra-Fernandez;Sabherwal (2001); Choo (2006); Takeuchi; Nonaka (2008); Oliveira et al. (2010); Baldam et al. (2014); ABPMP (2015).

$\mathrm{Na}$ construção do questionário utilizado na pesquisa, conforme preconiza Vieira (2009), considerou-se não apenas os objetivos do estudo, como também a tipificação dos respondentes. Dessa forma, a estrutura do questionário foi composta por questões sobre fatos (levantamento acerca dos próprios respondentes, no tocante à intimidade dos mesmos com o tema e à experiência profissional) e questões sobre opiniões (levantamento da percepção dos respondentes sobre a integração entre GC e BPM), essas últimas compostas por declarações com cinco 
alternativas de resposta, conhecidas como itens de escala Likert, graduadas de tal forma que o respondente, ao fazer a sua escolha, indicou seu posicionamento, sua concordância perante a pergunta feita (VIEIRA, 2009).

Após a definição da estrutura numérica do questionário e da elaboração das questões, promoveu-se um pré-teste com o propósito de validar o conteúdo textual, no tocante à associação das práticas de GC como apoiadoras das atividades de BPM. Tal validação foi feita por especialistas, voluntários do MBA em Gestão Estratégica de Processos de Negócio, do IEC PUC Minas.

A coleta de dados deu-se por meio do questionário definitivo, disponibilizado online, por 75 dias, para toda a população alvo da pesquisa, formada por 3.300 pessoas, e respondido por meio da autoaplicação, sendo obtidas 353 respostas.

No que concerne à análise dos dados da pesquisa, como destacado por Babbie (1999), o método survey, em geral, é combinado com o método estatístico para interpretação dos resultados. Neste sentido, para que fosse possível a organização, sumarização e descrição do conjunto de dados, aplicou-se a estatística descritiva, conforme indicado por Martins (2005). Para a análise estatística dos dados das questões sobre opiniões, optou-se pela estatística inferencial, posto que seu objetivo é obter informações sobre uma população a partir de resultados observados na amostra (COSTA, 2018).

Inicialmente, foram tabulados os dados inerentes ao perfil dos respondentes em relação à experiência dos mesmos em BPM, sendo excluídas as respostas advindas de participantes que informaram não ter experiência na área. Em seguida, por meio do software IBM SPSS 22 (StatisticalPackage for Social Science), foram realizadas as análises estatísticas das percepções dos respondentes sobre os construtos da pesquisa.

\section{Apresentação e Análise dos Resultados}

Nesta seção são expostas as principais análises dos resultados obtidos na pesquisa completa. Primeiramente, apresenta-se o perfil dos respondentes, seguido das observações sobre a consistência interna da escala utilizada no questionário, posto que o mesmo foi desenvolvido a partir do modelo conceitual da pesquisa. Na sequência, está a síntese dos resultados das análises estatísticas inferenciais, realizadas com base em testes não paramétricos das variáveis dos construtos do estudo.

\subsection{Perfil dos Respondentes}


No tocante ao perfil dos respondentes, foram levantadas informações sobre a atuação desses em atividades de BPM (tipo de engajamento, maturidade e região geográfica de atuação)e se o profissional era ou não certificado pela ABPMP.

Em termos do tipo de atuação em atividades de BPM, a amostra obtida foi composta por: 188 analistas ou consultores em BPM que atuam em diversas organizações $(54,49 \%) ; 82$ respondentes integrantes do escritório de processos da organização em que trabalham $(23,77 \%)$; e 75 profissionais que trabalham, em suas organizações, com foco em técnicas e atividades da metodologia BPM (21,74\%). O tempo de atuação dos respondentes está dividido da seguinte forma: 4,35\% dos profissionais atuam em BPM há menos de 1 ano (15 respondentes); 11,59\% desempenham atividades na área há mais de 1 ano (40 respondentes); e $84,06 \%$ atuam na área há mais de três anos (290 respondentes). No que se refere à certificação profissional, dos 345 participantes da pesquisa, 236 são CBPPs.

Os resultados obtidos, tanto em relação às atividades desempenhadas quanto ao tempo de atividade dos profissionais, refletem - público alvo da pesquisa. Posto que, como explicitado nos procedimentos de coleta de dados, 887 profissionais convidados a participar da pesquisa eram CBPPs, ou seja, especialistas em BPM que, para obter a certificação na área, fizeram uma prova para a avaliação dos seus conhecimentos sobre a metodologia e tiveram que comprovar, junto à ABPMP, um tempo mínimo de três anos experiência. Tal análise está, inclusive, apoiada pelo fato de $68 \%$ da amostra obtida ser composta por CBPPs.

No tocante à variável referente à região geográfica, verificou-se que pesquisa coletou percepções de profissionais atuantes em todo o território nacional. A Região Sudeste foi a mais marcada pelos respondentes (53,36\%), seguida pelas Regiões Centro-oeste $(19,06 \%)$ e Sul $(13,68 \%)$. Vale destacar que grande parcela da população alvo foi originada da rede de contatos da regional mineira da ABPMP, o que, muito provavelmente, impactou no volume de profissionais que participaram da pesquisa e atuam na Região Sudeste. Apesar da menor representatividade, o resultado das Regiões Nordeste $(10,54 \%)$ e Norte $(3,36 \%)$ corroboram a validade das conclusões deste estudo. Mesmo que em proporções diferentes, as percepções dos respondentes sobre práticas de GC como apoiadoras das atividades de BPM representam realidades diversas em todo país.

\subsection{Análise da Consistência da Escala Utilizada}

A análise da fidedignidade da escala do questionário para cada um dos 5 construtos (ou dimensões) que, em conjunto, representam a relação 
integrativa entre GC e BPM, de acordo com o modelo conceitual da pesquisa, foi realizada por meio do coeficiente Alfa de Cronbach, largamente utilizado pela academia. Esse coeficiente é uma medida de confiabilidade que varia de 0 a 1 , sendo os valores de 0,60 a 0,70 considerados o limite inferior de aceitabilidade, conforme Hairet al., (2009).

Por meio do SPSS, calculou-se o coeficiente Alta de Cronbach considerando os blocos das dimensões do instrumento de pesquisa. Por estarem todos acima do valor mínimo normalmente recomendado (isto é, acima de 0,70$)$, os resultados indicaram que o questionário aplicado era confiável. Na análise de confiabilidade realizada, também foi considerado o impacto da retirada de cada item para o coeficiente do bloco, sem que houvesse a verificação de melhoria significativa quantitativa no caso de exclusão de qualquer questão.

\subsection{Análises Estatísticas das Percepções dos Respondentes}

Após a realização de testes de normalidade para cada bloco, verificou-se que as distribuições dos conjuntos amostrais eram significativamente diferentes de uma distribuição normal, constatação cujo impacto resultou na necessidade de uso de testes não paramétricos na estatística inferencial (FIELD, 2009). A partir dos pressupostos da pesquisa, estruturados com base na contribuição do referencial teórico estudado, foram elaboradas hipóteses associadas ao objetivo da pesquisa.

Dessa forma, primeiramente, por meio do Teste de Friedman, foram analisadas as amostras relacionadas às respostas de todos os participantes para todas as questões do bloco respectivo, isto é, amostras pareadas. Então, por meio do Teste de Wilcoxon-Mann-Whitney, buscouse revelar, para cada bloco, diferenças significativas entre as respostas advindas de amostras independentes, formadas por dois grupos, CBPPs e não CBPPs.

A análise dos dados tornou possível a identificação da percepção dos respondentes sobre 0 apoio do Modelo SECI às atividades de gerenciamento de processos, bem como o levantamento das principais práticas que podem ser aplicadas para a conversão do conhecimento em cada fase do ciclo de atividades típicas de BPM, proposto pelo BPM CBOK. A Tabela 1 apresenta a síntese dos resultados e das análises, conforme os objetivos específicos da pesquisa. 
Tabela 1: Síntese dos resultados conforme objetivos da pesquisa

\begin{tabular}{|c|c|}
\hline Objetivos & $\begin{array}{c}\text { Análises a partir dos resultados do tratamento } \\
\text { estatístico }\end{array}$ \\
\hline $\begin{array}{l}\text { Levantar a percepção de } \\
\text { profissionais atuantes em } \\
\text { BPM sobre a integração } \\
\text { entre BPM e GC. }\end{array}$ & $\begin{array}{l}\text { - O Modelo SECI, na percepção dos respondentes, impacta de forma } \\
\text { diferente as atividades de BPM, sendo que tal percepção não } \\
\text { diferente entre os CBPPs e não CBPPs participantes da pesquisa. } \\
\text { - As percepções dos respondentes sobre o uso de práticas de } \\
\text { socialização, externalização, combinação e internalização diferem } \\
\text { entre as fases do Ciclo de vida BPM CBOK, o que credita } \\
\text { importância aos resultados obtidos para o cumprimento do segundo } \\
\text { objetivo específico. } \\
\text { - Nos testes comparativos entre os participantes CBPPs e não } \\
\text { CBPPs, verificou-se que, mesmo havendo diferença estatística na } \\
\text { percepção entre os pares em relação a determinadas práticas (S, E, } \\
\text { C, I), naquelas identificadas como mais frequentes, não houve } \\
\text { diferença estatística, corroborando os resultados obtidos para o } \\
\text { cumprimento do segundo objetivo específico. }\end{array}$ \\
\hline $\begin{array}{l}\text { Identificar, a partir das } \\
\text { experiências desses } \\
\text { profissionais, as práticas de } \\
\text { GC mais adotadas nas fases } \\
\text { do Ciclo de vida BPM } \\
\text { (proposto pelo CBOK): } \\
\text { planejamento, análise, } \\
\text { desenho, implementação, } \\
\text { monitoramento / controle e } \\
\text { refinamento. }\end{array}$ & $\begin{array}{l}\text { - No planejamento, a captura de conhecimento de especialistas e o } \\
\text { uso de sistemas de suporte à decisão são as práticas mais } \\
\text { frequentes. } \\
\text { - Na análise, a consulta a documentos e a observação da prática são } \\
\text { mais frequentes. } \\
\text { - No desenho, o uso de uma notação comum e a consulta à base de } \\
\text { dados da modelagem de processos são as práticas mais comuns. } \\
\text { - Na implementação, a consulta a manuais, o treinamento no posto } \\
\text { de trabalho e o aprendizado na prática são mais frequentes. } \\
\text { - No monitoramento \& controle, a explicitação clara de regras e } \\
\text { fluxos, bem como a consulta a documentos e o aprendizado por } \\
\text { observação são comuns. } \\
\text { - No refinamento, reuniões presenciais, histórias e relatos orais são } \\
\text { frequentes. }\end{array}$ \\
\hline
\end{tabular}

Fonte: Elaborado pelos autores (2019).

A Figura 4 apresenta, de forma gráfica, as práticas recomendadas na literatura em relação as quais os respondentes expressaram maior nível de concordância, ou seja, as práticas mais frequentemente aplicadas por tais profissionais nas atividades de BPM. 
Figura 4: Práticas do Modelo SECI mais utilizadas em BPM

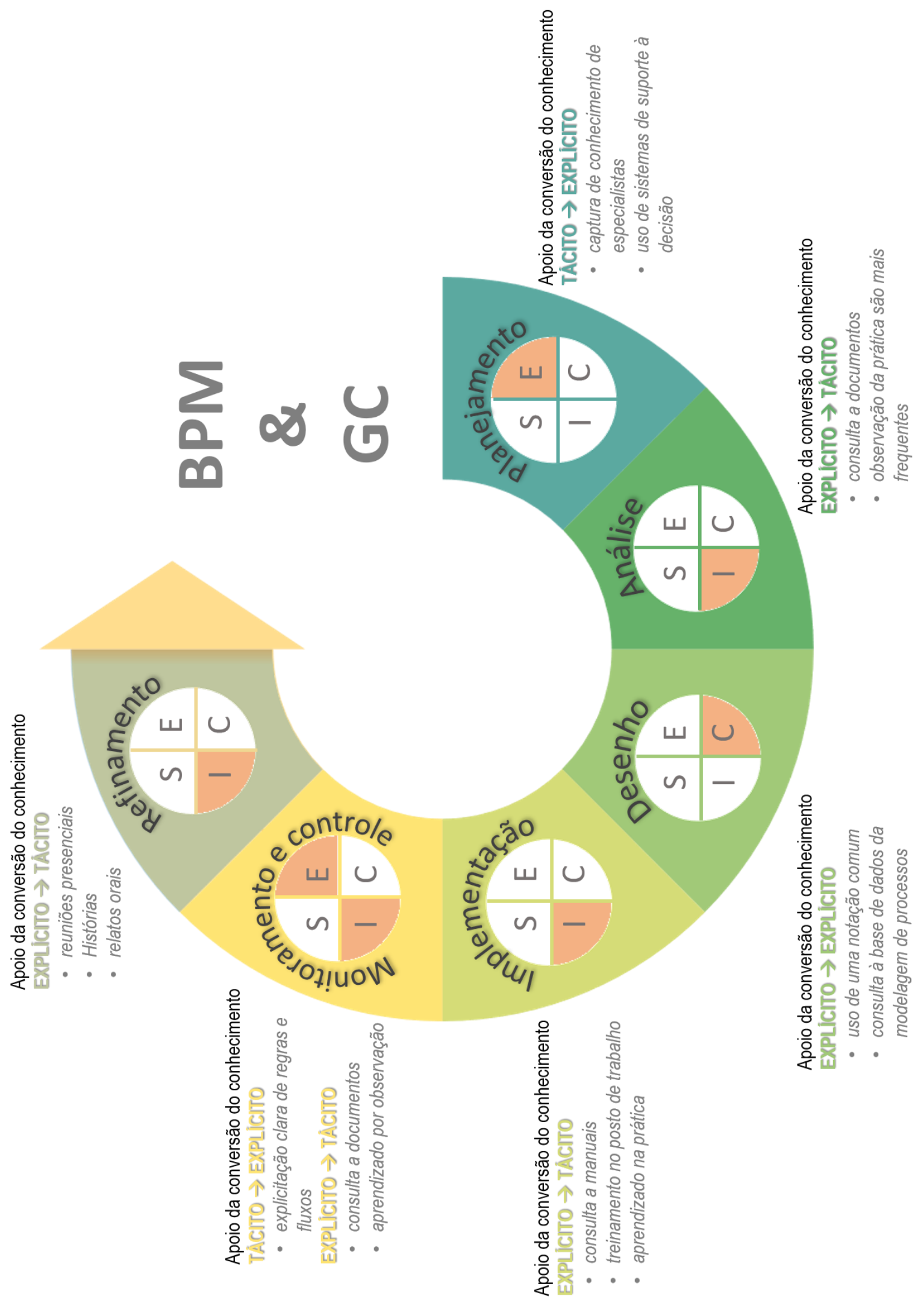

Fonte: Elaborado pelos autores (2019) 


\section{Discussão dos Resultados e Considerações Finais}

Este estudo teve como objetivo identificar de que forma a Gestão do Conhecimento pode contribuir como suporte ao Business Process Management, na percepção de profissionais que atuam com gerenciamento de processos de negócio. No tocante à relevância do tema, para Davenport (2013), é muito importante que as organizações procurem melhorar o trabalho por meio da gestão do conhecimento ou de iniciativas sistemáticas para aprimorar a distribuição e utilização do conhecimento.

Para ser possível a identificação de como a GC pode apoiar o BPM, o estudo abordou a relação integrativa entre tais áreas de conhecimento e, com base no levantamento teórico sobre os temas, optou-se por pesquisar o apoio do Modelo SECI (NONAKA; TAKEUCHI, 1997) às atividades do Ciclo de vida BPM, constante no CBOK (CommomBodyofKnowledge) da ABPMP (Associationof Business Process Management Professionals). Nessa perspectiva, portanto, a pesquisa teve como objetivo geral identificar de que forma a GC pode contribuir como suporte ao BPM, na percepção de profissionais que atuam com gerenciamento de processos de negócio. Para atingir esse objetivo foi realizada uma pesquisa descritiva, com abordagem quantitativa, do tipo survey, com profissionais atuantes em BPM.

O primeiro objetivo específico foi levantar a percepção de profissionais atuantes em gerenciamento de processos de negócio sobre a integração entre BPM e GC. Tal meta está alinhada com Davenport (2013), que assegura que melhorar o desempenho dos trabalhadores do conhecimento é bastante desafiador, mas carece de ferramentas e metodologias adequadas. Para tal, elaborou-se o modelo conceitual da pesquisa, utilizado no desenvolvimento do instrumento de coleta de dados e na definição dos pressupostos da pesquisa. A população alvo do estudo englobou todos os CBPPs do Brasil, bem como profissionais participantes de eventos sobre BPM realizados pela Regional Minas da ABPMP. Os dados coletados foram analisados por meio de técnicas estatísticas com vistas a avaliar a aceitação ou não dos pressupostos da pesquisa.

A análise global das respostas sobre questões de opiniões permitiu a verificação do pressuposto de que a GC contribui como suporte ao BPM em todas as fases do ciclo de vida proposto no CBOK. Todavia, de acordo com as percepções dos respondentes, de fato, o Modelo SECI impacta de forma diferente as atividades de BPM. Vale ressaltar, no entanto, que os resultados mostraram uma relevante concordância dos profissionais sobre a importância da GC em todas as fases, posto que não houve resultados de discordância em nenhum dos construtos avaliados. Tal verificação está apoiada por Hammer (2013) que destaca a importância de se aplicar a GC 
ao contexto dos processos de negócio da organização. Os resultados das análises, portanto, viabilizaram o cumprimento do primeiro objetivo específico ao proporcionarem um levantamento claro da percepção de profissionais em BPM sobre a relação integrativa entre BPM e GC.

O segundo objetivo específico englobou a identificação, a partir das experiências desses profissionais, das práticas de GC mais adotadas nas fases do Ciclo de vida BPM CBOK. A relevância desse levantamento é apoiada por Drucker (2000), que defende que organizações baseadas em conhecimento precisam de propósitos claros e comuns que possam ser traduzidos em ações específicas, como, por exemplo, as práticas de conversão de conhecimento pesquisadas nesta pesquisa. Assim sendo, para o cumprimento do segundo objetivo específico, foram investigadas na literatura as indicações de práticas para cada atividade do ciclo de vida típico de BPM (NONAKA, 2000; BECERRA-FERNANDEZ; SABHERWAL, 2001; CHOO, 2006; TAKEUCHI; NONAKA, 2008; OLIVEIRA et al., 2010). Os resultados obtidos foram explorados por meio de análises estatísticas descritivas, sendo possível a identificação das práticas de conversão de conhecimento que, na opinião dos respondentes, são mais frequentes nas atividades típicas de BPM. As práticas identificadas podem apoiar gestores no tocante à priorização e ao estímulo de determinadas práticas conforme os objetivos organizacionais de criação de conhecimento.

A partir do cumprimento dos objetivos específicos, conclui-se que o estudo respondeu à pergunta de partida ao identificar de que forma a GC pode contribuir como suporte ao BPM, na percepção de profissionais que atuam com gerenciamento de processos de negócio. Vale destacar que, por se tratar de uma pesquisa quantitativa, buscou-se fundamentar as análises em técnicas estatísticas, apoiadas nos conceitos teóricos pesquisados, objetivando levantar características da população a partir da amostra. Em relação aos respondentes, ressalta-se a significativa representatividade de profissionais certificados em BPM, consultores, assim como analistas de processos, atuantes em diferentes setores da economia, desempenhando atividades de BPM com certo nível de maturidade e advindos de todas as regiões do país. Tais aspectos corroboram as conclusões do estudo, que podem apoiar decisões de gestores no sentido de priorizar técnicas de GC que, de fato, promovam a criação do conhecimento e a melhoria no desempenho das atividades de BPM.

A proposta de desenvolvimento de um instrumento de coleta de dados como o utilizado nesta pesquisa, no entanto, é uma tarefa que envolve incertezas. Dessa forma, o estudo possui como limitação o condicionamento das conclusões à estrutura do questionário. Apesar de ter sido concebido com base em um modelo conceitual e de ter sido avaliado por especialistas, as questões do instrumento de coleta de dados 
podem ser refinadas em estudos futuros, com vistas a tornar seus enunciados mais diretos e focados, por exemplo, em uma única opção a ser avaliada sobre determinado construto ou dimensão da pesquisa.

Todavia, o questionário também representa uma das relevantes contribuições do estudo. O aprofundamento da investigação ora apresentada pode ocorrer por meio da adaptação do instrumento de coleta de dados para aplicação em pesquisas qualitativas, com vistas a levantar percepções mais adensadas sobre o apoio da GC às atividades de BPM. Neste sentido, o instrumento de coleta de dados pode ser adaptado como um roteiro de entrevistas ou na discussão em grupos focais, nos quais ocorram debates e compartilhamento de experiências de profissionais atuantes em BPM.

O diagrama proposto para a pesquisa, ao representar a relação integrativa entre BPM e GC, sob a ótica das atividades típicas propostas no BPM CBOK e as práticas do Modelo SECI, pode servir de base para investigações complementares sobre o tema ou para a aplicação em práticas de conversão do conhecimento. Isto é, o conhecimento explícito criado nesta pesquisa pode ser combinado e transformado em conhecimento organizacional, seja para a criação de diagramas adaptados a diferentes realidades organizacionais - combinação (explícito para explícito) - ou para a capacitação de colaboradores sobre o tema internalização (explícito para tácito).

Vale igualmente ressaltar o papel do modelo conceitual, estruturado a partir das principais contribuições do referencial teórico da pesquisa, que propiciou a criação do instrumento de pesquisa aplicado na coleta de dados. Além da síntese clara e objetiva das teorias dos autores, o modelo, que contempla também a representação das relações entre os conceitos e práticas do Modelo SECI e as atividades do Ciclo de Vida BPM CBOK, pode ser utilizado como plataforma investigativa, norteando pesquisas adicionais que possam ampliar o arcabouço teórico sobre o tema.

Os resultados desta pesquisa, bem como as análises estruturadas, podem, da mesma forma que o questionário, ser usados como base para pesquisas futuras que busquem validar as conclusões desta pesquisa, por exemplo, por meio de estudos de caso aplicados em organizações de diferentes portes, segmentos de mercado e com níveis diversos de maturidade em BPM.

Finalmente, por investigar a efetividade da relação integrativa entre BPM e GC, este estudo amplia a discussão sobre práticas que unam ambas as abordagens em prol dos objetivos organizacionais. Assim sendo, ao buscar desvelar aspectos dos processos de negócio e sua gestão, esta pesquisa é relevante no sentido de fornecer o contexto prático das atividades de GC, conforme destacado por Marjanovic e Freeze (2012). As percepções dos profissionais, analisadas no âmbito do modelo conceitual 
da pesquisa, contribuem também para a redução da lacuna, identificada por Van der Aalstet al. (2016), entre o uso real do BPM e o estado da arte das ferramentas e abordagens da metodologia.

\section{Referências}

ABPMP - Association of Business Process Management Professionals. BPM CBOK V3.0: guia para o gerenciamento de processos de negócio ( $3^{a}$ ed). ABPMP Brasil, 2015.

BABBIE, E. Métodos de Pesquisas de Survey. Belo Horizonte: UFMG, 1999.

BALDAM, R. de L.; VALLE, R.; ROZENFELD, H. Gerenciamento de processos de negócios - BPM: uma referência para implantação prática. Rio de Janeiro: Elsevier, 2014.

BEM, R. M. de;RIBEIRO JÚNIOR, D. I. A Gestão do Conhecimento dentro das organizações: a participação do bibliotecário. Revista ACB:

Biblioteconomia em Santa Catarina, v.11, n.1, p.75-82, 2006.

CONTADOR, J. C.; SORDI, J. O.; MARINHO, B. de L. ;CARVALHO, M. F. H. Gestão do Conhecimento aplicada à Gestão por Processos: identificação de funcionalidades requeridas às soluções de Business Process Management System (BPMS). Revista de Administração e Inovação- RAI, v.2, n. 2, p.5$18,2005$.

COSTA, G. G. Curso de Probabilidades e Estatística Inferencial: teoria e prática. 2.ed. Rio de Janeiro: Ciência Moderna, 2018.

DAVENPORT, T. H. Gestão de Processos no Trabalho do Conhecimento. In: BROCKE, J; ROSEMANN, M (Orgs). Manual de BPM: gestão de processos de negócio. Porto Alegre: Bookman, 2013.

DAVENPORT, T. H.; PRUSAK, L. Conhecimento empresarial: como as organizações gerenciam seu capital intelectual. Rio de Janeiro: Campus, 1998.

DRUCKER, P. F. O advento da nova organização. In: Gestão do conhecimento / Harvard Business Review. Rio de Janeiro: Campus, 2000.

FIELD, A. Discovering statistics using SPSS. 3.ed. London: Sage, 2009.

FREITAS, H. et al.O método de pesquisa survey. Revista de Administração da Universidade de São Paulo, v.35, n. 3, p.105-112, 2000.

HAIR, J. F.et al. Análise multivariada de dados. 6.ed. Porto Alegre: Bookman, 2009. 
HAMMER, M. O que é Gestão de Processos de Negócio? In: BROCKE, J; ROSEMANN, M (Orgs). Manual de BPM: gestão de processos de negócio. Porto Alegre: Bookman, 2013.

JUNG, J.; CHOI, I.; SONG, M. An integration architecture for knowledge systems and business process management systems. Computers in Industry, v.58, n.1, p.21-34, 2007.

KALPIČ, B.; BERNUS, P. Business process modeling through the knowledge management perspective. Journal of Knowledge Management, v.10, n.3, p.40-56, 2006.

MAFRA PEREIRA, F. C. Fontes de informação para negócios: análise sobre frequência, relevância e confiabilidade, baseada em estudo empírico com empresários e gestores organizacionais. Perspectivas em Ciência da Informação, v.21, n.2, p.100-119, 2016.

MALHOTRA, N. K. Pesquisa de Marketing: uma orientação aplicada. Porto Alegre: Bookman, 2012.

MARJANOVIC, O.; FREEZE, R. Knowledge-Intensive Business: deriving a sustainable competitive advantage through business process management and knowledge management integration. Knowledge and Process Management, v.19, n.4, p.180-188, 2012.

MARTINS, G. de A. Estatística Geral e Aplicada. 3.ed. São Paulo: Atlas, 2005.

MORENO, V.;DOS SANTOS, L. H. A. Gestão do conhecimento e redesenho de processos de negócio: proposta de uma metodologia integrada. Perspectivas em Ciência da Informação, v.17, n. 1, p.203-230, 2012.

NONAKA, I.;TAKEUCHI, H. Criação de conhecimento na empresa: como as empresas japonesas geram a dinâmica da inovação. Rio de Janeiro:

Elsevier, 1997.

OLIVEIRA, S. L. Tratado de metodologia científica: projetos de pesquisas, TGI, TCC, monografias, dissertações e teses. São Paulo: Pioneira, 2001.

RECORDS, L. R. The fusion of process and knowledge management. BP Trends, Sept., 2005.

ROBBINS, S. O comportamento organizacional. Rio de Janeiro: Livros Técnicos e Científicos, 1999.

SABBAG, P. Y. Espirais do conhecimento: ativando indivíduos, grupos e organizações. São Paulo: Saraiva, 2007. 
SENA, M. A. C. Processos de conhecimento como parte integrante dos processos de negócio: um modelo aplicado fundamentado na integração entre gestão do conhecimento e business process management. 2015. Tese (Doutorado) - Programa de Pós-Graduação em Engenharia e Gestão do Conhecimento. Universidade Federal de Santa Catarina, Curitiba, PR, 2013. Disponível em:

https://repositorio.ufsc.br/handle/123456789/135653. Acesso em 30 nov. 2018.

SILVA, A. C. L. BPM Business Process Management: introdução sobre BPM em uma visão integrada e didática para a gestão estratégica de processos de negócio. São Paulo: Bookess, 2017.

SORDI, J. O. Gestão por processos: uma abordagem da moderna administração. 5.ed. São Paulo: Saraiva, 2018.

VAN DER AALST, W. M. P. ; LA ROSA, M. ; SANTORO, F. M. BusinesS Process Management: don't forget to improve the process. Bus Inf Syst Eng, v.58, n.1, p.1-6, 2016.

VIEIRA, S. Como elaborar questionários. São Paulo: Atlas, 2009.

YAXIONG, T.et al. A research on BPM system based on process knowledge. In: IEEE CONFERENCE ON CYBERNETICS AND INTELLIGENT SYSTEMS. CHENGDU, 2008. 